Clinical Images

\title{
Cholecystitis caused by a fish bone
}

Masaki Kunizaki, M.D., Ph.D. ${ }^{a, b}$; Hiroyuki Kusano, M.D., Ph.D. ${ }^{\text {b }}$ Koji Azuma,

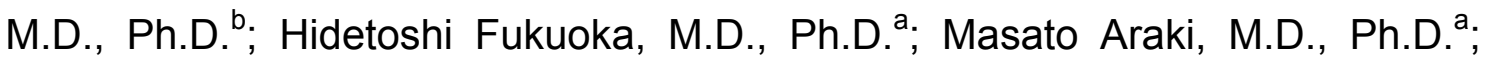
Shigekazu Hidaka, M.D., Ph.D. ${ }^{a}$; Atsushi Nanashima, M.D., Ph.D. ${ }^{\text {a.; }}$ Terumitu Sawai, M.D., Ph.D. a. Toru Yasutake, M.D., Ph.D. ${ }^{\text {a. }}$; and Takeshi Nagayasu, M.D., Ph.D. ${ }^{a}$

aDivision of Surgical Oncology, Department of Translational Medical Sciences, Nagasaki University Graduate School of Biomedical Sciences, 1-7-1 Sakamoto, Nagasaki 852-8501, Japan

${ }^{\mathrm{b}}$ Department of Surgery, Japan Seafarers Association Nagasaki Hospital, 5-16 Kabashima, Nagasaki 850-0034, Japan

Correspondence should be addressed to Masaki Kunizaki

Division of Surgical Oncology, Department of Translational Medical Sciences, Nagasaki University Graduate School of Biomedical Sciences, 1-7-1 Sakamoto, Nagasaki 852-8501, Japan

Tel: 095-819-7304; Fax: 095-819-7306

URL: http://www.med.nagasaki-u.ac.jp/surgery1/

E-mail: makuni49@nagasaki-u.ac.jp 


\section{Abstract}

The accidental ingestion of a foreign body is not uncommon. However, the presence of a foreign body in the gallbladder is extremely rare. Here, we present a case of cholecystitis due to a fish bone which may have penetrated through the stomach wall and into the gallbladder without causing peritonitis. The patient presented with right upper abdominal pain. Abdominal X-ray, ultrasonography and computed tomography findings suggested the presence of a foreign body resembling a fish bone in the gall bladder without evidence of free air or ascites. A laparoscopic cholecystectomy was performed; a fish bone, measuring $4.0 \mathrm{~cm}$ in length, was found in the gallbladder. Microscopic examination revealed granulation of the gallbladder wall with fibrosis. The postoperative course was uneventful. To the best of our knowledge, this is the first such case to be reported. 
A 67-year-old man presented with a 3-week history of a dull, upper abdominal pain that had persisted despite medication. He had no other significant medical history. Physical examination revealed abdominal tenderness and distension, but no mass was felt. Laboratory findings revealed a white blood cell count of $5400 / \mathrm{mm}^{3}$ and a C-reactive protein value of $0.30 \mathrm{mg} / \mathrm{dl}$ (normal: $<0.5 \mathrm{mg} / \mathrm{dl}$ ). Other laboratory findings were within normal limits. Plain abdominal X-ray film showed a linear calcified lesion in the right upper abdomen. Abdominal ultrasonography showed a linear hyperechoic lesion in the gallbladder (Fig. 1). In addition, computed tomography (CT) demonstrated a linear calcified lesion in the gallbladder and a small calcified lesion in the stomach wall. No hepatic duct dilatation, biliary abnormalities or abscess formation were noted (Fig. 2). No granuloma or fistula was seen by upper gastroduodenal endoscopy. Further questioning of the patient revealed that he habitually ate his favorite fish without removal of the bones. The history combined with the radiological findings suggested that a fish bone may have penetrated the stomach wall and into the gallbladder without causing overt peritonitis. A laparoscopic cholecystectomy was performed. Some adhesions were noted around the gallbladder 
but there was no communication between the gallbladder and the gastrointestinal tract. On gross examination, a fish bone, measuring $4.0 \mathrm{~cm}$ in length, was present inside the gallbladder (Fig. 3). The mucosal surface revealed granulation of the gallbladder wall with fibrosis. Histopathological findings revealed mild chronic cholecystitis and fibrous scar with tissue eosinophilia. The patient had an uneventful postoperative course and was discharged eight days after the procedure. 


\section{Comments}

While most ingested foreign bodies pass through the gastrointestinal tract uneventfully within a week, approximately $1 \%$ require surgical removal. Chu et al. reported that fish bones are the most common foreign bodies causing gastrointestinal tract perforation in Hong Kong ${ }^{1}$. Foreign bodies in the hepatobiliary system commonly consist of residual objects from a previous operation and food materials. Ingested foreign material in the biliary tree is usually associated with a previous biliary tree operation ${ }^{2,3}$. Foreign bodies in the hepatobiliary system are a rare cause of biliary stones. Prochazka et al. reported two cases of choledocholithiasis caused by foreign material in patients who had not undergone any prior operation ${ }^{4}$. They concluded that the foreign material within the stones in patients without an operative history may be due to reflux from the duodenum. In the present case, we believe that it was unlikely that the fish bone reached the gallbladder as a result of duodenal reflux through the plica spiralis. Despite the fact that there was no demonstrable choledochoenteric fistula, we speculate that the fish bone may have penetrated through the 
stomach wall and into the gallbladder without causing overt peritonitis. Recently, the laparoscopic approach has been increasingly recognized as a procedure offering precise, visual assessment of the intra-abdominal condition, allowing accurate diagnosis, prompt treatment, and further examination. We must not forget the importance of making a preoperative diagnosis before complications develop, because perforation may occur following the ingestion of a sharp, pointed foreign body. Ultrasonography, CT and/or endoscopic investigations should be performed, and appropriate management, including laparoscopic intervention, should be provided. 


\section{References}

1. Chu KM, Choi HK, Tuen $\mathrm{HH}$, et al. A prospective randomized trial comparing the use of the flexible gastroscope versus the bronchoscope in the management of foreign body ingestion. Gastrointest Endosc 1998;47:23-7.

2. George B, John KS, Lloyd MN, et al. The behavior of foreign bodies in the extrahepatic billiary system. Am J Surg 1964;108:250-6.

3. George LF. Foreign body in the abdomen causing obstruction. Am J Surg 1953;86:472-5.

4. Prochazka V, Krausova D, Kod'ousek R, et al. Foreign material as a cause of choledocholithiasis. Endoscopy 1999;31:383-5. 


\section{Figure legends}

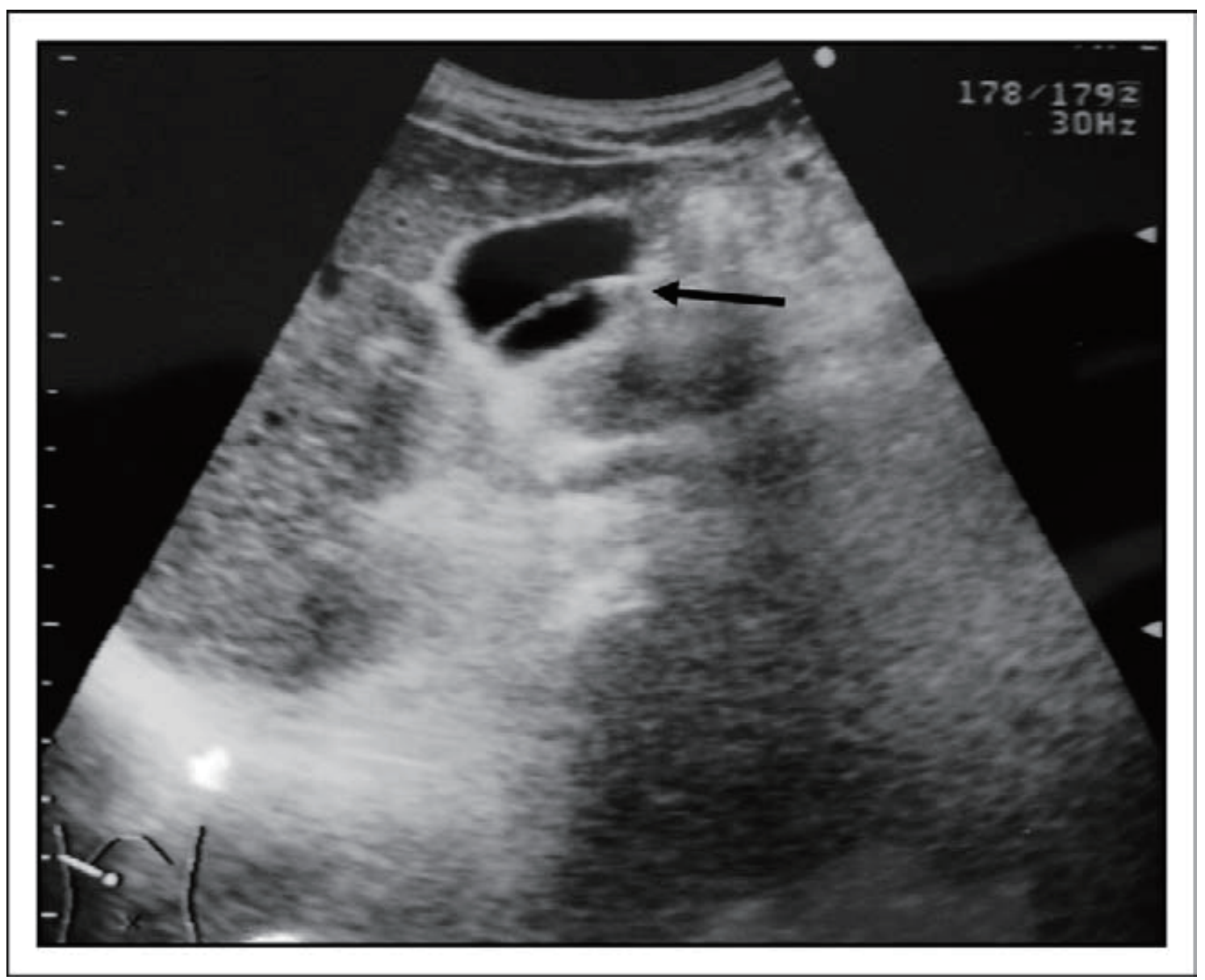

Figure 1. Ultrasonography revealed a linear hyperechoic lesion in the gallbladder. 


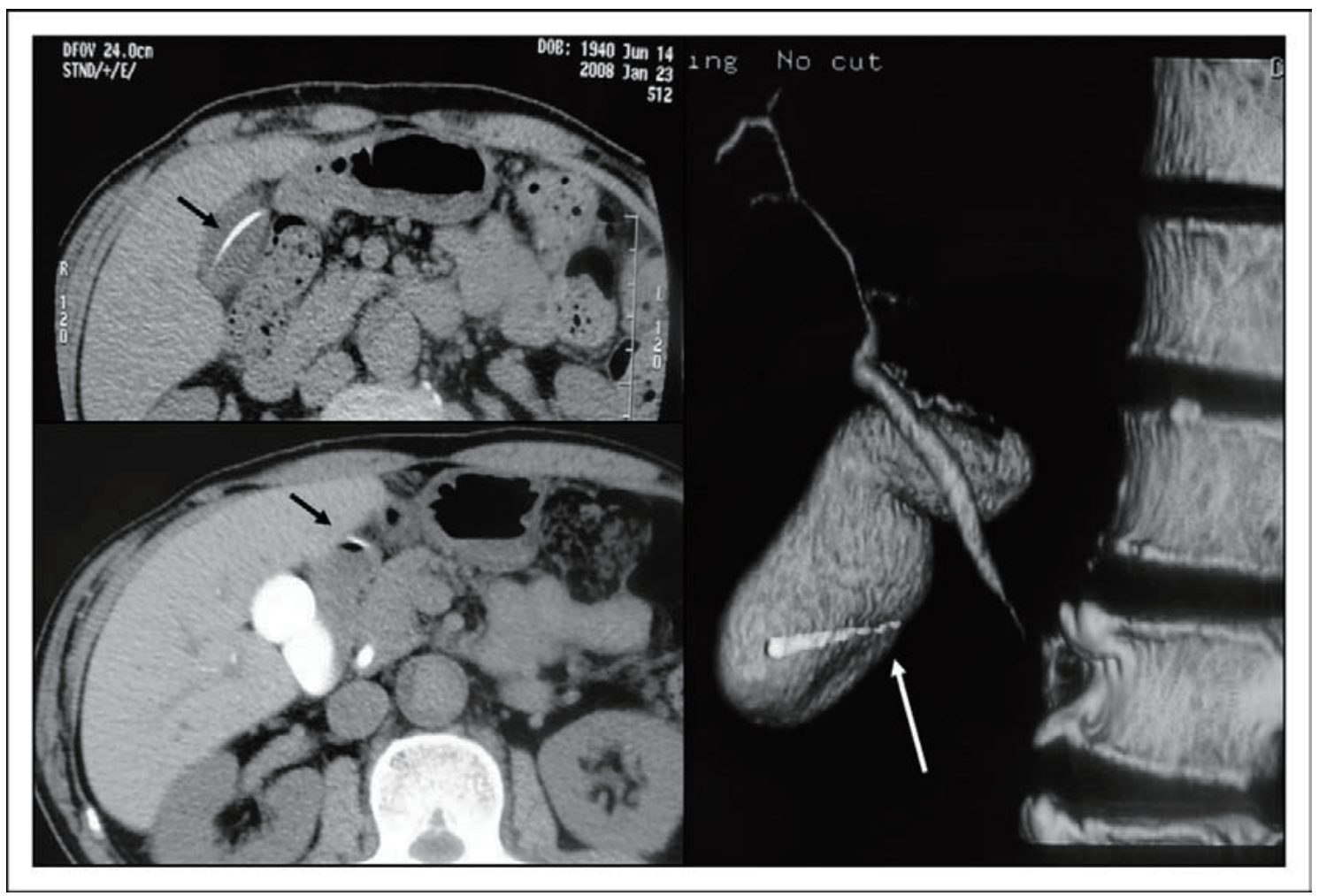

Figure 2. Plain CT scan showed a linear radiopaque density in the gallbladder.

Preoperative drip infusion cholangiography CT scan showed a fish bone in the gallbladder and a small linear density in the gastric wall without surrounding inflammation. 


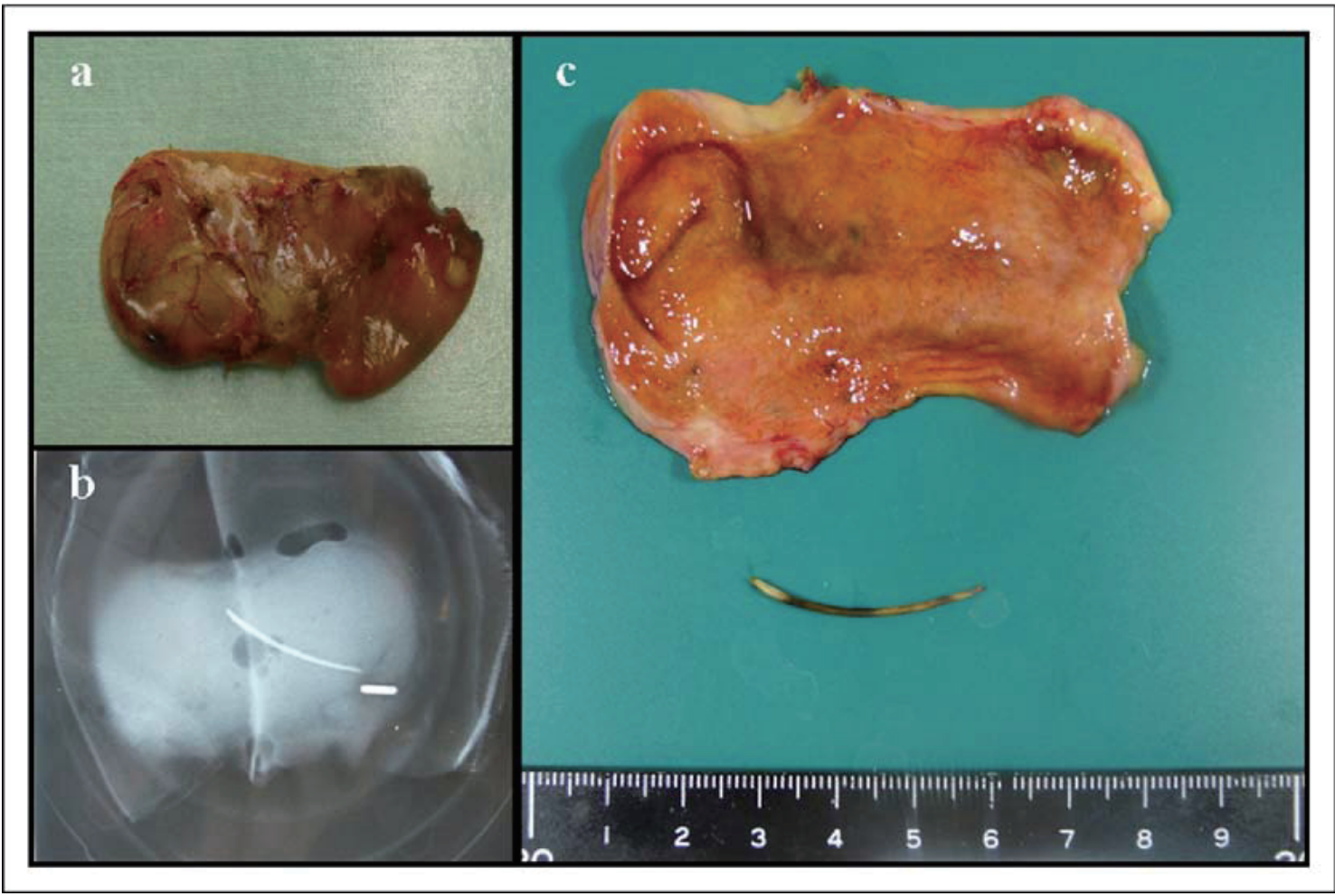

Figure 3. (A) Surgical specimen of the gallbladder. (B) A specimen radiograph showing a fish bone in the gallbladder. (C) A fishbone, about $4.0 \mathrm{~cm}$ in size, was extracted from the gallbladder. 